\title{
GAS5-mediated regulation of cell signaling (Review)
}

\author{
YANG ZHOU and BINGHAI CHEN \\ Department of Urology, Affiliated Hospital of Jiangsu University, Zhenjiang, Jiangsu 212000, P.R. China
}

Received February 15, 2020; Accepted June 19, 2020

DOI: $10.3892 / \mathrm{mmr} .2020 .11435$

\begin{abstract}
In recent years, an increasing number of long non-coding RNAs (lncRNAs) have been discovered using microarrays and nucleic acid sequencing technology. LncRNAs exert crucial biological functions by regulating signaling pathways. In particular, the lncRNA growth arrest-specific transcript 5 (GAS5) has been documented to serve a crucial role in numerous signaling pathways. This article discusses the latest developments in the association between GAS5 and microRNA (miRNA), p53, mTOR, glucocorticoid response element (GRE) and AKT in order to investigate the roles served by GAS5. miRNAs can activate related signaling pathways and GAS5 can combine with miRNA to regulate related signaling pathways. GAS5 may regulate p53 expression via derivation of snoRNA, but the underlying mechanism requires further investigation. GAS5 overxpresion reduces the expression level of mTOR, which is induced by inhibiting miR-106a-5p expression. GAS5 is a sponge of GR, and serves a role in controlling and maintaining glucocorticoid sensitivity and drug resistance via competitive combination with GR. GAS5 can interact with miRNAs, such as miR-21 and miR-532-5p, to regulate the expression of AKT signaling pathway, affecting cell survival and apoptosis. Collectively, the data indicate that GAS5 serves a key role in the miRNA, p53, mTOR, GRE, and AKT signaling pathways. GAS5 regulates complex intracellular signaling pathways primarily through three modes of action, all of which are interrelated: Signal, decoy and guide. In the present article, latest developments in the association between GAS5 and a number of cellular signaling pathways are discussed to examine the tumor suppressive role of GAS5.
\end{abstract}

Correspondence to: Dr Binghai Chen, Department of Urology, Affiliated Hospital of Jiangsu University, 438 Jiefang Road, Zhenjiang, Jiangsu 212000, P.R. China

E-mail: chenbhny@163.com

Abbreviations: lncRNAs, long noncoding RNAs; GAS5, growth arrest-specific transcript 5; PTEN, phosphatase and tensin homologue deleted on chromosome 10; PDCD4, programmed cell death 4; GR, glucocorticoid receptor; GRE, glucocorticoid response element

Key words: long non-coding RNAs, growth arrest-specific transcript 5, cellular signaling pathway

\section{Contents}

1. Introduction

2. Four modes of action by which GAS5 regulates signaling pathways

3. Regulation of gene expression by GAS5

4. GAS5 and the p53 network

5. GAS5 and mTOR

6. GAS5 and microRNA (miR or miRNA)

7. GAS5 and glucocorticoid response element (GRE)

8. GAS5 and AKT

9. Conclusion

\section{Introduction}

Although previous studies have discovered numerous RNA species, protein coding RNAs account for only the minority of the transcriptome $(1,2)$. The majority of RNA transcripts are actually comprised of non-coding RNAs $(1,2)$. Noncoding RNA can be divided into two categories according to nucleotide (nt) length: i) Long non-coding RNA (lncRNA; >200 nt); and ii) short noncoding RNA (<200 nt) (3). The majority of previous studies initially considered lncRNA to be a type of transcriptional noise (4). However, further research over the past decade revealed that lncRNAs serve an important roles in a wide of range of biological functions, including cell growth and developmental processes (5). There are several methods used for classifying lncRNAs, one of which categorizes lncRNAs based on their position relative to protein-coding genes: i) Intergenic lncRNA; ii) intron lncRNA; iii) overlapping lncRNA; and iv) antisense lncRNA (6). Currently, although the regulation of gene expression of short non-coding RNAs, such miRNAs, has been relatively well studied, reports on the effects of lncRNAs on gene expression remain insufficient (7). However, numerous previous studies have indicated that lncRNAs serve a variety of roles in regulating cell signaling, such as the JAK/STAT, PI3K/Akt and Wnt signaling pathway (8-10).

A well-known lncRNA, growth arrest-specific transcript (GAS) 5, has recently become the focus of research attention. The GAS1-5 paralogs constitute the GAS multigene family (11). GAS1-5 proteins share a common $\mathrm{N}$-terminal domain but exhibit different C-terminal extensions (12). GAS5a and GAS5b are produced by the alternative splicing of 12 exons at the 1q25 locus in the GAS5 gene (13). Previous studies have reported that GAS5 serves an anti-cancer effect in various types of cancer, including gastric cancer (14), lung cancer (15), 
hepatocellular carcinoma (16) and renal cancer (17). The accumulation of GAS5 has been found to inhibit cancer growth, invasion and metastasis, and enhance cell sensitivity to chemotherapy drugs (18-21). Since elucidating the underlying molecular mechanism of GAS5 function is becoming a subject of intense investigation, the present review aims to discuss the current knowledge on the GAS5-mediated regulation of cell signaling (Table I).

\section{Three modes of action by which GAS5 regulates signaling pathways}

There are three modes of action by which GAS5 participates in signaling pathways. Firstly, GAS5 can serve as a signaling protein, where it is specifically transcribed following different triggers and then participates in signal transmission in a number of pathways as a signaling node (22). For instance, experiments have indicated that after DNA damage in human colorectal tissue cells, GAS5 increases the expression of snoRNA, and GAS5 snoRNA activates the p53 signaling pathway and responds to DNA damage. GAS5 serves as a signaling protein in the aforementioned process and directly participates in the regulation of p53 signaling pathway (23). Secondly, GAS5 can act as a decoy, where it can behave as a molecular sponge that binds directly to target RNA or proteins, thereby blocking their downstream functions (24). For example, GAS5 binds to miR-21 to prevent its inhibitory action on PTEN and PDCD4, thereby upregulating the expression of PTEN and PDCD4 (25). Thirdly, GAS5 can serve a guide role as a transport protein, where upon binding, it guides the protein to a specific DNA sequence where it can regulate the transcription of downstream molecules (26). GAS5 has been previously reported to promote the binding of E2F transcription factor 1 (E2F1) to the promoter of the cyclin-dependent kinase inhibitor 1B (P27kip1) gene through guidance to activate P27kip1 (27) (Fig. 1).

\section{Regulation of gene expression by GAS5}

LncRNAs can directly interact with promoters to modulate transcription (28). GAS5 binds to elements, leading to the activation or inhibition of gene expression (28). GAS5 can indirectly activate $\mathrm{P} 27 \mathrm{kip} 1$ transcription by facilitating the binding of E2F1 to the P27kip1 promoter, by directly binding to E2F1 itself (Fig. 2A) (29). In addition, GAS5 has been documented to inhibit the translation of c-Myc mRNA by interacting with the eukaryotic translation initiation factor 4E, without affecting the transcription or protein stability of c-Myc mRNA 18). In gastric cancer, Y-box binding protein 1 (YBX1) has been confirmed to interact with GAS5. Downregulation of GAS5 expression reduces the expression level of $\mathrm{YBX} 1$ and further reduces the expression of p21 induced by YBX1 (30) GAS5 has been demonstrated to enhance lung cancer cell apoptosis induced by gefitinib, a selective epidermal growth factor receptor tyrosine kinase inhibitor, by inhibiting epidermal growth factor and downregulating the expression of insulin-like growth factor receptor 1 (31). Furthermore, GAS5 overexpression in cells following myocardial ischemia-reperfusion injury activates the p38 MAPK signaling pathway and increases the expression of LAS1 to promote apoptosis (32). Chemokine (C-C motif) ligand 1 overexpression in bladder cancer cells can also be induced by reducing the expression of GAS5 (33).

GAS5 is involved in PTEN regulation through a number of mechanisms. PTEN is an important tumor suppressor that serves an important role in cell growth, apoptosis and migration (34). GAS5 may indirectly facilitate the translation of PTEN by repressing miR-21a-5p (35). Additionally, in papillary thyroid carcinoma, GAS5 can serve as a molecular sponge for miR-222-3p, which activates the PTEN/AKT pathway (36). Similarly, GAS5 has been found to sponge miR-222 to indirectly suppress PTEN expression in breast cancer (37).

\section{GAS5 and the p53 network}

p53 is an extensively studied tumor suppressor gene that regulates cell cycle progression and prevents carcinogenesis (38). p53 is therefore termed the 'guardian of the genome' (39). The predominant role of p53 is to maintain genome stability and avoid mutation. A number of lncRNAs have been found to regulate the p53 network $(40,41)$. Previous studies have indicated that reduced GAS5 expression is associated with cell cycle arrest via increased p53 expression, breast cancer gene type 1 and growth damage and DNA damage inducible $\alpha$ expression (40-42). In colorectal cancer cell lines, p53 expression has been previously found to associate closely with GAS5-derived snoRNA levels (23). Therefore, GAS5 may regulate p53 expression via a feedback loop, the mechanism of which requires further investigation. The feedback pathway is activated after DNA damage as GAS5 derives snoRNA, which directly promotes the expression of $\mathrm{p} 53$ and inhibits the $\mathrm{G}_{1}$ phase in the middle stage of cell division, which inhibits DNA damage in cells undergoing mitosis (Fig. 2B).

\section{GAS5 and mTOR}

mTOR is involved in numerous signaling pathways, including cell proliferation, differentiation, autophagy and apoptosis (43). A regulatory relationship between mTOR and GAS5 has been previously found $(44,45)$. Several studies have demonstrated that GAS5 is expressed at reduced levels in gastric cancer cells $(46,47)$. GAS5 overexpression has been revealed to inhibit the proliferation of gastric cancer cells by reducing mTOR expression, suggesting that GAS5 exerts its biological function through the mTOR pathway (48). One study reported that the expression level of GAS5 in gastric cancer is negatively associated with that of miR-106a-5p. Overexpression of miR-106a-5p reverses the biological effects induced by GAS5 overexpression, which occurs via Akt/mTOR (49). This shows that GAS5 overexpression serves a role in inhibiting proliferation in gastric cancer cells by inhibiting the function of miR-106a-5p, thereby reducing mTOR expression (49). In patients with esophageal cancer, GAS5 expression has been demonstrated to be reduced. In esophageal cancer cell lines, GAS5 overexpression has been found to inhibit proliferation, migration, reduce PI3K expression and AKT and mTOR phosphorylation. The use of PI3K agonists reversed these aforementioned effects without affecting the expression of GAS5 $(50,51)$. This suggests that GAS5 mediates its effects through the PI3K/mTOR pathway in esophageal cancer cells $(50,51)$. Additionally, a previous study on glioma cells revealed that GAS5 inhibits excessive 
Table I. GAS5 involved signal pathways and corresponding regulatory genes.

\begin{tabular}{lll}
\hline Role of GAS5 & \multicolumn{1}{c}{ Gene involved } & \multicolumn{1}{c}{ References } \\
\hline $\begin{array}{l}\text { Regulation } \\
\text { of gene }\end{array}$ & p27kip1, E2F1 & Luo et al $(27)$ \\
expression & c-Myc & Hu et al $(18)$ \\
& EGFR & Liu et al $(30)$ \\
& p38, LAS1 & Dong et al $(31)$ \\
p53 network & CCL1 & Liu et al $(32)$ \\
& BRCA1, GADD45A & Cao et al $(33)$ \\
mTOR & Mazar et al $(40)$ \\
& miRNA-106a-5p & Krell et al $(23)$ \\
miRNA & PI3K & Dong et al $(49)$ \\
& miR-21, PDCD4, & Wang et al $(50)$ \\
& miR-103 & \\
& Crhr1, miR449a et al $(25)$ \\
& miR-21a-5p & Nemoto et al $(62)$ \\
& miR-222-3p & Gao et al $(35)$ \\
miR-222 & Zhang et al $(36)$ \\
GRE & miR-532-5p & Gu et al $(37)$ \\
AKT & cIAP2 and SGK1 & Han et al $(76)$ \\
& MMP-9 and TP53 & Lucafo et al $(65)$ \\
& ROCK1 & Zheng et al $(75)$ \\
& & Wu et al $(78)$
\end{tabular}

GAS5, growth arrest-specific 5 .

autophagy induced by cisplatin exposure by activating the mTOR signaling pathway (52). It was found that treatment with rapamycin (mTOR antagonist) can eliminate GAS5-mediated inhibition of autophagy caused by cisplatin and reduce GAS5 sensitivity to cisplatin (52).

\section{GAS5 and miRNA}

miRNAs are a class of short-sequence RNAs that are abundant in eukaryotes (53). Although miRNAs have been found to regulate the expression of numerous protein-coding genes, whether miRNAs target lncRNAs remains unclear (54). Previous studies have shown that GAS5 is closely related to multiple miRNAs (55-57). For example, previous studies have demonstrated a feedback pathway between GAS5 and miR-21, where GAS5 induces a reduction in miR-21 expression to activate the translation of crucial genes, including PTEN and PDCD4 $(58,59)$. Additionally, GAS5 has been found to bind with miR-103 to upregulate PTEN expression (25) (Fig. 3A).

In hepatocellular carcinoma (HCC) tissues, GAS5, PDCD4 and PTEN levels were reduced whilst miR-21 levels were increased compared with those in adjacent normal tissue (60). Hepatocellular carcinoma (HCC) is a common tumor with high malignancy and poor prognosis. Furthermore, the expression levels of GAS5 and miR-21 are associated with the clinical features of HCC (60). Lower GAS5 expression levels are correlated with higher miR-21 expression levels (60). Compared with patients with liver cancer with low GAS5 expression levels, patients with liver cancer with high GAS5 expression levels survive longer (60). GAS5 acts as a negative regulator in liver cancer through miR-21 and its targets, which are involved in the migration and invasion of cancer cells (61). This indicates that GAS5 may be a target for the treatment of HCC (61). Additionally, increased expression of GAS5 may lead to increased corticotropin-releasing hormone receptor 1 expression and long-term activation of the hypothalamic-pituitary-adrenal axis, possibly due to the inhibition of miR449a by GAS5 (62).

\section{GAS5 and glucocorticoid response element (GRE)}

Glucocorticoid receptors (GRs) are important members of the nuclear-receptor family and common transcription regulators depending on hormone levels (63). Following hormone binding, GRs regulate the expression of target genes, including apoptosis genes cIAP2 and SGK1, by interacting with the glucocorticoid response element (GRE) (64). Via the aforementioned signaling pathways, GR can regulate the process of cell apoptosis. GAS5 and GR interact as GAS5 is considered to be a sponge for GR, suggesting that GAS5 is a possible factor for the control and maintenance of glucocorticoid sensitivity and drug resistance (65). Previous studies have demonstrated that GAS5 competes with GRE to bind the DNA-binding domain (DBD) sites on GRs $(66,67)$. This process blocks GR binding to the GRE, consequently repressing the transcriptional activation of target genes (Fig. 3B) (68).

\section{GAS5 and AKT}

Activation of the AKT pathway occurs through a variety of signaling pathways, including those of MAPK, PI3K/AKT and mTOR. The AKT pathway is closely associated with the proliferative and invasive abilities of tumor cells. GAS5 serves an important role in the regulation of the AKT pathway $(69,70)$. Previous studies have reported that GAS5 interacts with the PDCD4 protein (71-73). In cardiomyocytes, GAS5 competitively binds to miR-21, thereby positively regulating PDCD4 expression. The enhanced expression of PDCD4 in turn inhibits the PI3K/AKT signaling pathway (74). This indicates that GAS5 regulates the expression of PDCD4 by targeting miR-21 and indirectly inhibiting the PI3K/AKT signaling pathway (74). Inhibiting the expression of GAS5 activates the PI3K/AKT signaling pathway and increases the expression of its downstream proteins matrix metallopeptidase 9 and tumor protein p53 in human trophoblast cell lines HTR-8/SVneo and JEG-3 (75). GAS5 competitively binds to miR-532-5p such that silencing GAS5 expression upregulates the expression of miR-532-5p to activate the PI3K/AKT signaling pathway in myocardial ischemia reperfusion injury (76). This indicates that GAS5 regulates the PI3K/AKT signaling pathway by binding with miR-532-5p (76). Furthermore, in ischaemic brain injury, GAS5 enhances the expression level of PTEN by regulating the expression of miR-21, thereby inhibiting the PI3K/AKT signaling pathway (77). In cardiomyocytes, GAS5 knockdown has been found to suppress the expression of Rho associated coiled-coil containing protein kinase 1 (ROCK1), activating the PI3K/AKT signaling pathway, which in turn suppress the expression of glycogen synthase kinase $3 \beta$ (GSK-3 $\beta$ ). miR-335 can bind to GAS5 and inhibit its expression. When miR-335 is inhibited, 


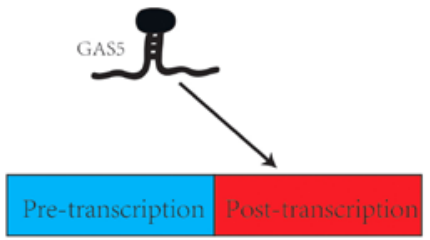

Signal

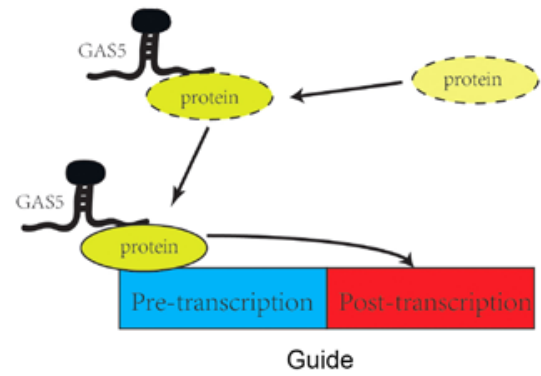

Guide

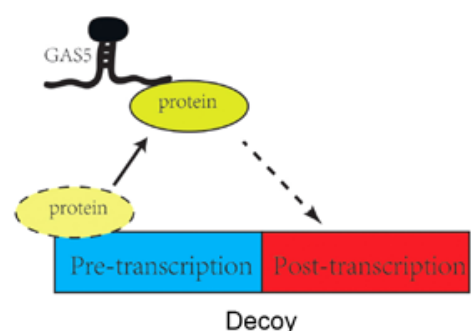

Figure 1. Three main modes of GAS5 participation in signaling pathways. GAS5 participates in complex signaling pathways mainly through three modes of action: Signal, sponge and guide. The line representations are as follows: (A) Solid, direct effect; (B) dotted, indirect effect; (C) solid ellipse, the final position of the protein; and (D) dotted ellipse, the original position of the protein or the original position in the pathway. GAS5, growth arrest-specific 5.

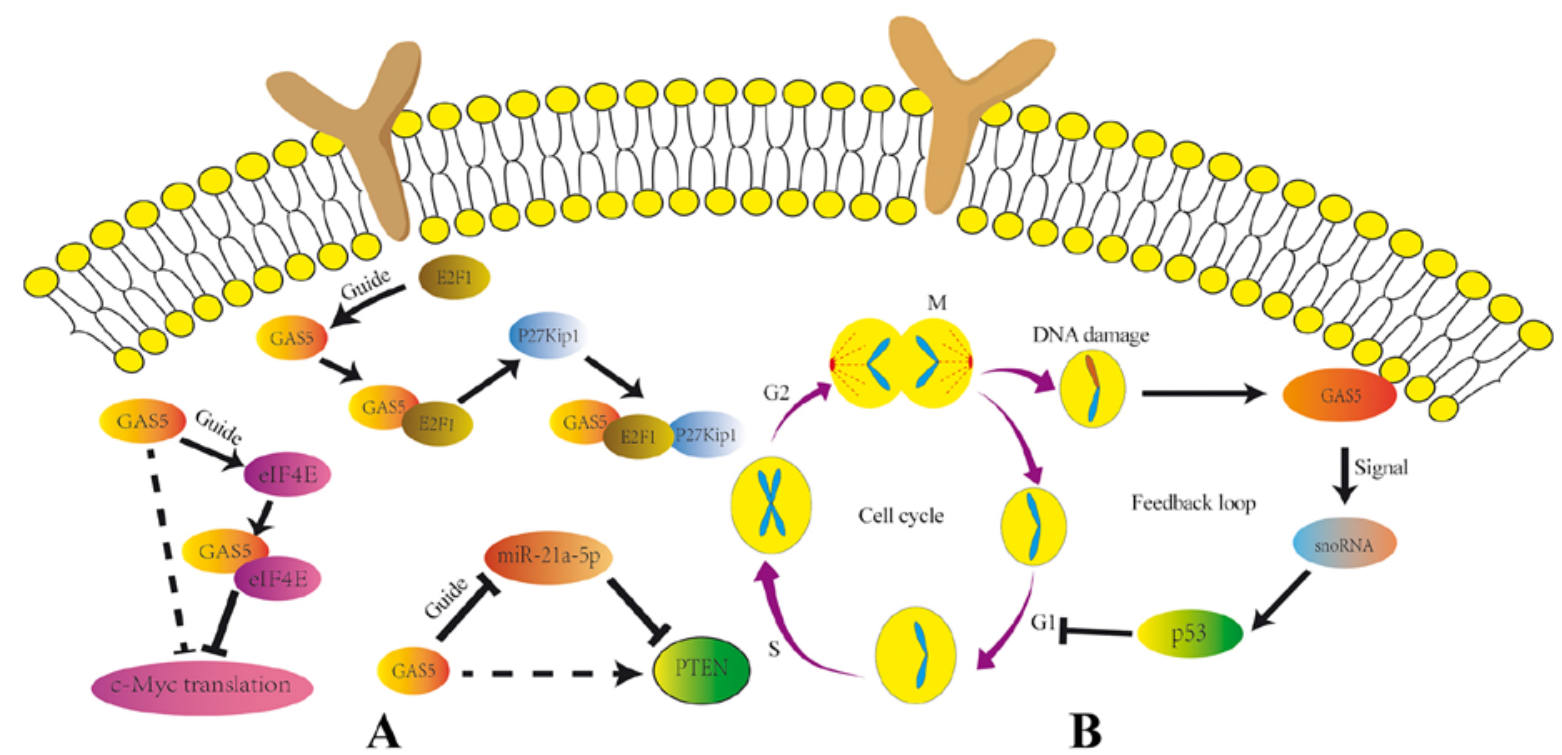

Figure 2. GAS5 regulates gene expression, and the relationship between GAS5 and p53 network. (A) Regulation of gene expression by lncRNA GAS5. GAS5 regulates the expression level of genes and enhances the interaction of E2F1 and P27kip1 by activating E2F1. GAS5 binds to eIF4E to inhibit c-Myc mRNA expression. GAS5 indirectly enhances the expression of PTEN by directly inhibiting the expression of miR-21a-5p. (B) GAS5 and the p53 network. Feedback adjustment of lncRNA GAS5 and p53. Following DNA damage, GAS5 can derive snoRNA, which directly promotes the expression of p53 and inhibits the G1 phase in the interphase of cell division. Solid edges represent direct regulatory effects. Dotted edges represent indirect regulatory effects. lncRNA, long noncoding RNA; GAS5, growth arrest-specific 5; E2F1, E2F transcription factor 1; P27kip1; cyclin-dependent kinase inhibitor 1B; eIF4E, eukaryotic translation initiation factor 4E; PTEN, phosphatase and tensin homologue deleted on chromosome 10; miR, microRNA; snoRNA, small nucleolar RNA.

the expression of GAS5 is increased and the aforementioned effects are abolished (78). This suggests that GAS5 exerts its biological function through the miR-335/ROCK1/AKT/GSK-3 $\beta$ axis (78). Furthermore, under hypoxic conditions, cell survival rate is reduced whereas apoptosis ability is enhanced. GAS5 expression in myocardial cells is increased and the PI3K/AKT signaling pathway is suppressed. Inhibiting the expression of GAS5 improves the viability of cells and inhibits cell apoptosis. Additionally, the expression of miR-222-3p, a target gene of
GAS5, was found to be downregulated under hypoxic conditions (79). Overexpression of miR-222-3p reversed the effects of GAS5 on cell survival and apoptosis under hypoxic conditions by activating the PI3K/AKT signaling pathway (79).

\section{Conclusions}

In short, GAS5 exerts its functions via three modes of action, namely signal, decoy and guide. In lung cancer, GAS5 can 


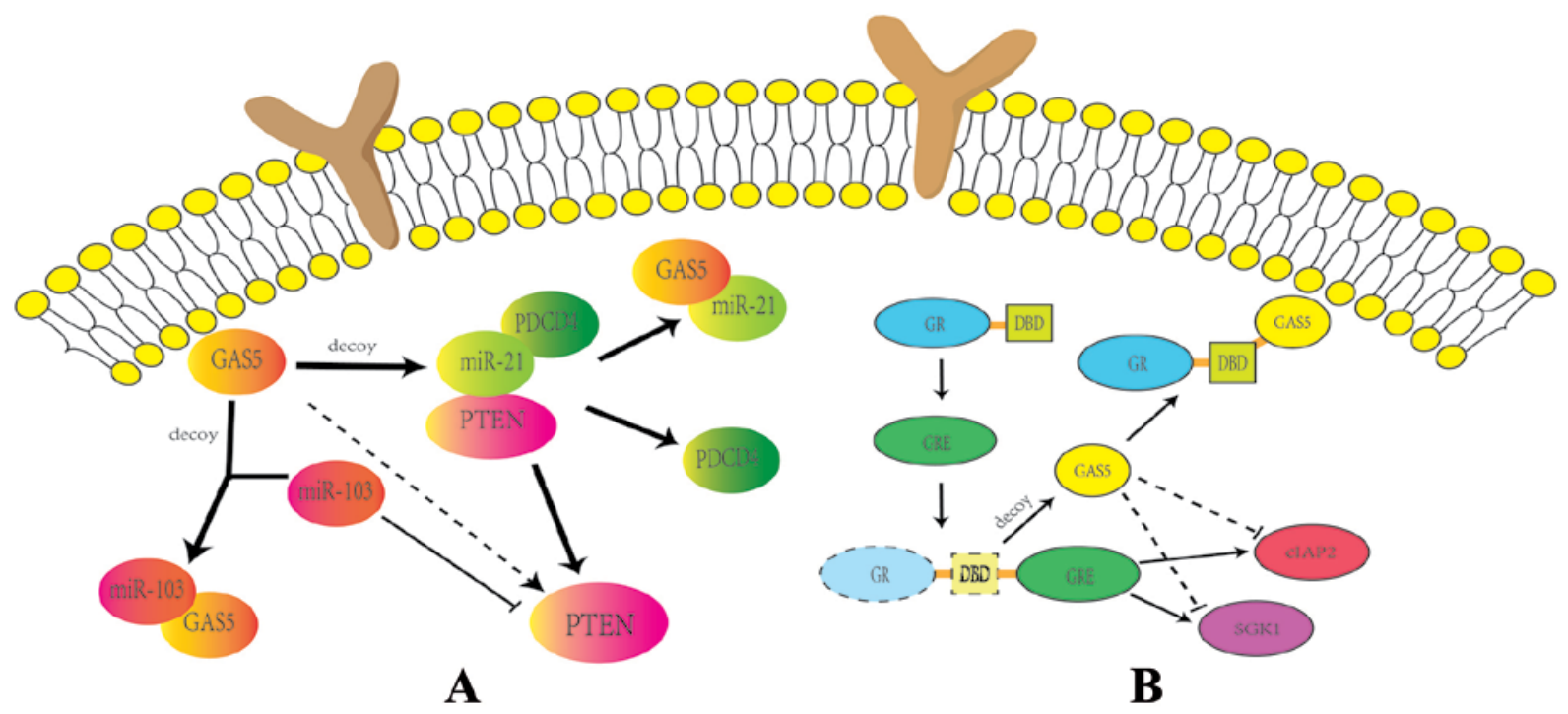

Figure 3. Relationship between GAS5 and miRNA, and the relationship between GAS5 and GRE. (A) The interaction of 1ncRNA GAS5 with miRNA. GAS5 activates PTEN, PDCD4 and miR-21 conjugates through competitive binding with miR-21. Additionally, GAS5 can directly bind to miR-103 to upregulate PTEN expression. (B) The association between IncRNA GAS5 and GRE. GAS5 competitively binds the DBD on the GR, preventing the binding of GR and GRE. Furthermore, GAS5 indirectly inhibits the expression of GR target genes cIAP2 and SGK1. Solid edges represent direct regulatory effects. Dotted edges represent indirect regulatory effects. IncRNA, long noncoding RNA; GAS5, growth arrest-specific 5; miRNA, microRNA; PTEN, phosphatase and tensin homologue deleted on chromosome 10; PDCD4, programmed cell death 4; GRE, glucocorticoid response element; DBD, DNA-binding domain; GR, glucocorticoid receptor; cIAP2, baculoviral IAP repeat-containing protein 3; SGK1, serum/glucocorticoid regulated kinase 1.

enhance the effect of gefitinib by inhibiting epidermal growth factor and downregulating the expression of insulin-like growth factor receptor 1 (22). GAS5 is underexpressed in esophageal cancer cells, and overexpression of GAS5 inhibits the proliferation and migration of tumor cells, and inhibits the expression of PI3K and mTOR signaling pathways (35). In hepatocellular carcinoma, GAS5 is involved in downregulating the invasion and migration of liver cancer cells by interacting with miR-21, and the level of GAS5 is positively correlated with the survival rate of patients with liver cancer (39). In myocardial ischemia-reperfusion injury, GAS5 can combine with miR-532-5p to inhibit the PI3K/AKT signaling pathway, and silencing GAS5 expression can upregulate miR-532-5p to activate the PI3K/AKT signaling pathway to reduce myocardial damage (47). GAS5 can act as a sponge for GR to block the GR/GRE/cIAP2 and SGK1 signaling pathways, and achieve the effect of controlling and maintaining glucocorticoid sensitivity and drug resistance (43).

GAS5 serves a key role in various signaling pathways and has a role in regulating gene expression. For example, GAS5 can act as a guide to regulate the translation of P27Kip1,PTEN and c-Myc whilst regulating the expression of p53 by acting as a signaling molecule to form a feedback loop (18). GAS5 expression is closely associated with the degree of mTOR expression. Overexpression of GAS5 reduces the expression of mTOR and miRNA-106a-5p in a PI3K-dependent manner (34). Furthermore, GAS5 interacts with miRNA primarily through sponging. In this process, GAS5 compete to bind to a key gene or protein in a pathway to alter their expression levels (37). Additionally, GAS5 can serve as a molecular sponge in the GRE pathway, where it competes with GRE for the DBD structure on GRs (43). GAS5 is also involved in the regulation of AKT, since GAS5 inhibition activates the AKT signaling pathway (45). Furthermore, PDCD4, miR-532-5p and ROCK1 all serve important roles in the regulation process of this pathway. Increasing the expression of GAS5 commonly inhibits the development of tumors and enhances the therapeutic effect of drugs on tumor cells.

In conclusion, these data indicate GAS5 serves key roles in miRNA, p53, mTOR, GRE and AKT signaling pathways. However, the mechanism of action of GAS5 remains unclear and further experiments are required to elucidate its functions. In gastric cancer, lung cancer, hepatocellular carcinoma and renal cancer, the role of GAS5 is similar to that of tumor suppressor genes. However, whether it is expressed at low levels only in these specific diseases or expressed at low levels in all diseases requires further research. Fortunately, there are continuous novel developments in this field.

\section{Acknowledgements}

Thanks to Mr. Wei Hu (Department of Andrology, The First Affiliated Hospital of University of South China) for assisting with the preparation of the current review.

\section{Funding}

The present study was supported by the National Natural Science Foundation of China (grant no. 81402100), the Foundation for 'Young Medical Talents' of Jiangsu Province (grant no. QNRC2016840), the 'Six Talent Peaks Project' in Jiangsu Province (grant no. WSW-007) and the Natural Science Foundation of Hunan Province (grant no. 2019JJ40269).

\section{Availability of data and materials}

Data sharing is not applicable to this article, as no datasets were generated or analyzed during the current study. 


\section{Authors' contributions}

YZ completed the majority of the current review, including the preparation of the manuscript. BC provided specific academic guidance and assisted in improving the manuscript. All authors read and approved the final manuscript.

\section{Ethics approval and consent to participate}

Not applicable.

\section{Patient consent for publication}

Not applicable.

\section{Competing interests}

The authors declare that they have no competing interests.

\section{References}

1. Philippe N, Bou Samra E, Boureux A, Mancheron A, Rufflé F, Bai Q, De Vos J, Rivals E and Commes T: Combining DGE and RNA-sequencing data to identify new polyA+ non-coding transcripts in the human genome. Nucleic Acids Res 42: 2820-2832, 2014.

2. Pauli A, Valen E and Schier AF: Identifying (non-)coding RNAs and small peptides: Challenges and opportunities. Bioessays 37 103-112, 2015

3. Shi Q and Yang X: Circulating MicroRNA and long noncoding RNA as biomarkers of cardiovascular diseases. J Cell Physiol 231: 751-755, 2016.

4. Xu S, Gong Y, Yin Y, Xing $\mathrm{H}$ and Zhang N: The multiple function of long noncoding RNAs in osteosarcoma progression, drug resistance and prognosis. Biomed Pharmacother 127: 110141, 2020.

5. Ricciuti B, Mencaroni C, Paglialunga L, Paciullo F, Crinò L, Chiari R and Metro G: Long noncoding RNAs: New insights into non-small cell lung cancer biology, diagnosis and therapy. Med Oncol 33: 18, 2016.

6. Yousefi H, Maheronnaghsh M, Molaei F, Mashouri L, Reza Aref A, Momeny M and Alahari SK: Long noncoding RNAs and exosomal lncRNAs: Classification, and mechanisms in breast cancer metastasis and drug resistance. Oncogene 39: 953-974, 2020.

7. Panir K, Schjenken JE, Robertson SA and Hull ML: Non-coding RNAs in endometriosis: A narrative review. Hum Reprod Update 24: 497-515, 2018.

8. Yang DW, Zhang X, Qian GB, Jiang MJ, Wang P and Wang KZ: Downregulation of long noncoding RNA LOC101928134 inhibits the synovial hyperplasia and cartilage destruction of osteoarthritis rats through the activation of the Janus $\mathrm{kinase/signal} \mathrm{transducers} \mathrm{and} \mathrm{activators} \mathrm{of} \mathrm{transcription}$ signaling pathway by upregulating IFNA1. J Cell Physiol 234 10523-10534, 2019.

9. Wu Q and Yi X: Down-regulation of long noncoding RNA MALAT1 protects hippocampal neurons against excessive autophagy and apoptosis via the PI3K/Akt Signaling Pathway in rats with epilepsy. J Mol Neurosci 65: 234-245, 2018

10. Zhang L, Fang Y, Cheng X, Lian YJ and Xu HL: Silencing of long noncoding RNA SOX21-AS1 relieves neuronal oxidative stress injury in mice with Alzheimer's disease by upregulating FZD3/5 via the Wnt Signaling Pathway. Mol Neurobiol 56: 3522-3537, 2019

11. Rolli E, Ragni E, de Medina-Redondo M, Arroyo J, de Aldana CR and Popolo L: Expression, stability, and replacement of glucan-remodeling enzymes during developmental transitions in Saccharomyces cerevisiae. Mol Biol Cell 22: 1585-1598, 2011.

12. Ragni E, Fontaine T, Gissi C, Latgè JP and Popolo L: The Gas family of proteins of Saccharomyces cerevisiae: Characterization and evolutionary analysis. Yeast 24: 297-308, 2007.
13. Schneider C, King RM and Philipson L: Genes specifically expressed at growth arrest of mammalian cells. Cell 54: 787-793, 1988.

14. Dong X, Gao W, Lv X, Wang Y, Wu Q, Yang Z, Mao G and Xing W: Association between lncRNA GAS5, MEG3, and PCAT-1 polymorphisms and cancer risk: A Meta-Analysis. Dis Markers 2020: 6723487, 2020.

15. Shi X, Sun M, Liu H, Yao Y, Kong R, Chen F and Song Y: A critical role for the long non-coding RNA GAS5 in proliferation and apoptosis in non-small-cell lung cancer. Mol Carcinogen 54 (Suppl 1): E1-E12, 2015.

16. Zhao P, Cui X, Zhao L, Liu L and Wang D: Overexpression of Growth-Arrest-Specific Transcript 5 improved cisplatin sensitivity in hepatocellular carcinoma through Sponging miR-222. DNA Cell Biol 39: 724-732, 2020.

17. Yang W, Zhang K, Li L, Ma K, Hong B, Gong Y and Gong K: Discovery and validation of the prognostic value of the lncRNAs encoding snoRNAs in patients with clear cell renal cell carcinoma. Aging (Albany NY) 12: 4424-4444, 2020.

18. Hu G, Lou Z and Gupta M: The long non-coding RNA GAS5 cooperates with the eukaryotic translation initiation factor 4E to regulate c-Myc translation. PLoS One 9: e107016, 2014.

19. Wu Y, Zhang L, Wang Y, Li H, Ren X, Wei F, Yu W, Wang X, Zhang L, Yu J and Hao X: Long noncoding RNA HOTAIR involvement in cancer. Tumor Biol 35: 9531-9538, 2014.

20. Yan L, Zhou J, Gao Y Ghazal S, Lu L, Bellone S, Yang Y, Liu N, Zhao X, Santin AD, et al: Regulation of tumor cell migration and invasion by the H19/let-7 axis is antagonized by metformin-induced DNA methylation. Oncogene 34: 3076-3084, 2015.

21. Li S, Wang Q, Qiang Q, Shan H, Shi M, Chen B, Zhao S and Yuan L: Sp1-mediated transcriptional regulation of MALAT1 plays a critical role in tumor. J Cancer Res Clin Oncol 141: 1909-1920, 2015.

22. Jiang L, Wang C and Shen X: lncRNA GAS5 suppresses ER stress-induced apoptosis and inflammation by regulating SERCA2b in HG-treated retinal epithelial cell. Mol Med Rep 22: 1072-1080, 2020.

23. Krell J, Frampton AE, Mirnezami R, Harding V, De Giorgio A, Roca Alonso L, Cohen P, Ottaviani S, Colombo T, Jacob J, et al: Growth arrest-specific transcript 5 associated snoRNA levels are related to p53 expression and DNA damage in colorectal cancer. PLoS One 9: e98561, 2014.

24. Qian X, Xu C, Zhao P and Qi Z: Long non-coding RNA GAS5 inhibited hepatitis $C$ virus replication by binding viral NS3 protein. Virology 492: 155-165, 2016.

25. Zhang Z, Zhu Z, Watabe K, Zhang X, Bai C, Xu M, Wu F and Mo YY: Negative regulation of lncRNA GAS5 by miR-21. Cell Death Differ 20: 1558-1568, 2013.

26. Liu X, Jiao T, Wang Y, Su W, Tang Z and Han C: Long non-coding RNA GAS5 acts as a molecular sponge to regulate miR-23a in gastric cancer. Minerva Med 2016 (Epub sahead of print).

27. Luo G, Liu D, Huang C, Wang M, Xiao X, Zeng F, Wang L and Jiang G: LncRNA GAS5 inhibits cellular proliferation by targeting P27 ${ }^{\text {Kip1 }}$. Mol Cancer Res 15: 789-799, 2017.

28. Eilebrecht S, Wilhelm E, Benecke BJ, Bell B and Benecke AG: HMGA1 directly interacts with TAR to modulate basal and Tat-dependent HIV transcription. RNA Biol 10: 436-444, 2013.

29. Aminian K, Mashayekhi F, Mirzanejad L and Salehi Z: A functional genetic variant in GAS5 IncRNA (rs145204276) modulates $\mathrm{p} 27^{\mathrm{Kip} 1}$ expression and confers risk for gastric cancer. Br J Biomed Sci 76: 83-85, 2019.

30. Liu Y, Zhao J, Zhang W, Gan J, Hu C, Huang G and Zhang Y: lncRNA GAS5 enhances G1 cell cycle arrest via binding to YBX1 to regulate $\mathrm{p} 21$ expression in stomach cancer. Sci Rep 5: 10159, 2015.

31. Dong S, Qu X, Li W, Zhong X, Li P, Yang S, Chen X, Shao M and Zhang L: The long non-coding RNA, GAS5, enhances gefitinib-induced cell death in innate EGFR tyrosine kinase inhibitor-resistant lung adenocarcinoma cells with wide-type EGFR via downregulation of the IGF-1R expression. J Hematol Oncol 8: 43, 2015.

32. Liu SD, Meng WX, Xu L, Chi C, Sun X and Liu HY: GAS5 promotes myocardial apoptosis in myocardial ischemia-reperfusion injury via upregulating LAS1 expression. Eur Rev Med Pharmacol Sci 22: 8447-8453, 2018. 
33. Cao Q, Wang N, Qi J, Gu Z and Shen H: Long non-coding RNA-GAS5 acts as a tumor suppressor in bladder transitional cell carcinoma via regulation of chemokine (C-C motif) ligand 1 expression. Mol Med Rep 13: 27-34, 2016.

34. Xing Y, Lin NU, Maurer MA, Chen H, Mahvash A, Sahin A Akcakanat A, Li Y, Abramson V, Litton J, et al: Phase II trial of AKT inhibitor MK-2206 in patients with advanced breast cancer who have tumors with PIK3CA or AKT mutations, and/or PTEN loss/PTEN mutation. Breast Cancer Res 21: 78, 2019.

35. Gao ZQ, Wang JF, Chen DH, Ma XS, Wu Y, Tang Z and Dang XW: Long non-coding RNA GAS5 suppresses pancreatic cancer metastasis through modulating miR-32-5p/PTEN axis. Cell Biosci 7: 66, 2017.

36. Zhang XF, Ye Y and Zhao SJ: lncRNA Gas5 acts as a ceRNA to regulate PTEN expression by sponging miR-222-3p in papillary thyroid carcinoma. Oncotarget 9: 3519-3530, 2017.

37. Gu J, Wang Y, Wang X, Zhou D, Shao C, Zhou M and He Z: Downregulation of lncRNA GAS5 confers tamoxifen resistance by activating miR-222 in breast cancer. Cancer Lett 434: 1-10, 2018.

38. de Oliveira GAP, Petronilho EC, Pedrote MM, Marques MA, Vieira TCRG, Cino EA and Silva JL: The status of p53 oligomeric and aggregation states in cancer. Biomolecules 10: 548, 2020.

39. Silva JL, Lima CGS, Rangel LP, Ferretti GDS, Pauli FP, Ribeiro RCB, da Silva TB, da Silva FC and Ferreira VF: Recent synthetic approaches towards small molecule reactivators of $\mathrm{p} 53$ Biomolecules 10: 635, 2020.

40. Mazar J, Rosado A, Shelley J, Marchica J and Westmoreland TJ: The long non-coding RNA GAS5 differentially regulates cell cycle arrest and apoptosis through activation of BRCA1 and p53 in human neuroblastoma. Oncotarget 8: 6589-6607, 2017.

41. Dong P, Xiong Y, Yue J, J B Hanley S, Kobayashi N, Todo Y and Watari H: Exploring lncRNA-Mediated regulatory networks in endometrial cancer cells and the tumor microenvironment: Advances and challenges. Cancers (Basel) 11: 234, 2019.

42. Ye D, Bao Z, Yu Y, Han Z, Yu Y, Xu Z, Ma W, Yuan Y, Zhang L, $\mathrm{Xu} \mathrm{Y}$, et al: Inhibition of cardiomyocyte differentiation of human induced pluripotent stem cells by Ribavirin: Implication for its cardiac developmental toxicity. Toxicology 435: 152422, 2020.

43. Zou Z, Tao T, Li H and Zhu X: mTOR signaling pathway and mTOR inhibitors in cancer: Progress and challenges. Cell Biosci 10: 31, 2020.

44. Li H, Liu Y, Huang J, Liu Y and Zhu Y: Association of genetic variants in lncRNA GAS5/miR-21/mTOR axis with risk and prognosis of coronary artery disease among a Chinese population. J Clin Lab Anal: e23430, 2020 (Epub ahead of print).

45. Yacqub-Usman K, Pickard MR and Williams GT: Reciprocal regulation of GAS5 lncRNA levels and mTOR inhibitor action in prostate cancer cells. Prostate 75: 693-705, 2015.

46. Esfandi F, Salehnezhad T, Taheri M, Afsharpad M, Hafez AA, Oskooei VK and Ghafouri-Fard S: Expression assessment of a panel of long non-coding RNAs in gastric malignancy. Exp Mol Pathol 113: 104383, 2020.

47. Zhang N, Wang AY, Wang XK, Sun XM and Xue HZ: GAS5 is downregulated in gastric cancer cells by promoter hypermethylation and regulates adriamycin sensitivity. Eur Rev Med Pharmacol Sci 20: 3199-3205, 2016

48. Li Y, Gu J and Lu H: The GAS5/miR-222 axis regulates proliferation of gastric cancer cells through the PTEN/Akt/mTOR Pathway. Dig Dis Sci 62: 3426-3437, 2017.

49. Dong S, Zhang X and Liu D: Overexpression of long noncoding RNA GAS5 suppresses tumorigenesis and development of gastric cancer by sponging miR-106a-5p through the Akt/mTOR pathway. Biol Open 8: bio041343, 2019.

50. Wang G, Sun J, Zhao H and Li H: Long Non-Coding RNA (lncRNA) Growth Arrest Specific 5 (GAS5) suppresses esophageal squamous cell carcinoma cell proliferation and migration by inactivating phosphatidylinositol 3-kinase (PI3K)/AKT/Mammalian target of rapamycin (mTOR) signaling pathway. Med Sci Monit 24: 7689-7696, 2018.

51. Huang J, Li Y, Lu Z, Che Y, Sun S, Mao S, Lei Y, Zang R, Li N, Sun N and He J: Long non-coding RNA GAS5 is induced by interferons and plays an antitumor role in esophageal squamous cell carcinoma. Cancer Med 7: 3157-3167, 2018.

52. Huo JF and Chen XB: Long noncoding RNA growth arrest-specific 5 facilitates glioma cell sensitivity to cisplatin by suppressing excessive autophagy in an mTOR-dependent manner. J Cell Biochem 120: 6127-6136, 2019.
53. Rustagi Y, Jaiswal HK, Rawal K, Kundu GC and Rani V: Comparative characterization of cardiac development specific microRNAs: Fetal regulators for future. PLoS One 10: e0139359, 2015.

54. Witwer KW and Halushka MK: Toward the promise of microRNAs-Enhancing reproducibility and rigor in microRNA research. RNA Biol 13: 1103-1116, 2016.

55. Shen X, Zhang Y, Wu X, Guo Y, Shi W, Qi J, Cong H, Wang X, $\mathrm{Wu} \mathrm{X}$ and Ju S: Upregulated lncRNA-PCAT1 is closely related to clinical diagnosis of multiple myeloma as a predictive biomarker in serum. Cancer Biomark 18: 257-263, 2017.

56. Jing Z, Gao L, Wang H, Chen J, Nie B and Hong Q: Long non-coding RNA GAS5 regulates human B lymphocytic leukaemia tumourigenesis and metastasis by sponging miR-222. Cancer Biomark 26: 385-392, 2019.

57. Wang W, Jia YJ, Yang YL, Xue M, Zheng ZJ, Wang L and Xue YM: lncRNA GAS5 exacerbates renal tubular epithelial fibrosis by acting as a competing endogenous RNA of miR-96-5p. Biomed Pharmacother 121: 109411, 2020.

58. Chen D, Guo Y, Chen Y, Guo Q, Chen J, Li Y, Zheng Q, Jiang M, $\mathrm{Xi} \mathrm{M}$ and Cheng L: lncRNA growth arrest-specific transcript 5 targets miR-21 gene and regulates bladder cancer cell proliferation and apoptosis through PTEN. Cancer Med 9: 2846-2858, 2020.

59. Lyu K, Xu Y, Yue H, Li Y, Zhao J, Chen L, Wu J, Zhu X, Chai L, Li C, et al: Long noncoding RNA GAS5 acts as a tumor suppressor in laryngeal squamous cell carcinoma Via miR-21. Cancer Manag Res 11: 8487-8498, 2019.

60. Tu ZQ, Li RJ, Mei JZ and Li XH: Down-regulation of long non-coding RNA GAS5 is associated with the prognosis of hepatocellular carcinoma. Int J Clin Exp Pathol 7: 4303-4309, 2014.

61. Hu L, Ye H, Huang G, Luo F, Liu Y, Liu Y, Yang X, Shen J, Liu Q and Zhang J: Long noncoding RNA GAS5 suppresses the migration and invasion of hepatocellular carcinoma cells via miR-21. Tumor Biol 37: 2691-2702, 2016.

62. Nemoto $\mathrm{T}$, Kakinuma $\mathrm{Y}$ and Shibasaki $\mathrm{T}$ : Impaired miR449a-induced downregulation of Crhr1 expression in low-birth-weight rats. J Endocrinol 224: 195-203, 2015.

63. Weikum ER, Okafor CD, D'Agostino EH, Colucci JK and Ortlund EA: Structural analysis of the glucocorticoid receptor ligand-binding domain in complex with triamcinolone acetonide and a fragment of the atypical coregulator, small heterodimer partner. Mol Pharmacol 92: 12-21, 2017.

64. Tani H, Torimura M and Akimitsu N: The RNA degradation pathway regulates the function of GAS5 a non-coding RNA in mammalian cells. PLoS One 8: e55684, 2013.

65. Lucafo M, De Iudicibus S, Di Silvestre A, Pelin M, Candussio L, Martelossi S, Tommasini A, Piscianz E, Ventura A and Decorti G: Long noncoding RNA GAS5: A novel marker involved in glucocorticoid response. Curr Mol Med 15: 94-99, 2015.

66. Gharesouran J, Taheri M, Sayad A, Ghafouri-Fard S, Mazdeh M and Omrani MD: The growth arrest-specific transcript 5 (GAS5) and nuclear receptor subfamily 3 Group C Member 1 (NR3C1): Novel markers involved in multiple sclerosis. Int J Mol Cell Med 7: 102-110, 2018.

67. Ketab FNG, Gharesouran J, Ghafouri-Fard S, Dastar S, Mazraeh SA, Hosseinzadeh H, Moradi M, Javadlar M, Hiradfar A, Rezamand A, et al: Dual biomarkers long non-coding RNA GAS5 and its target, NR3C1, contribute to acute myeloid leukemia. Exp Mol. Pathol 114: 104399, 2020.

68. Kino T, Hurt DE, Ichijo T, Nader N and Chrousos GP: Noncoding RNA gas 5 is a growth arrest- and starvation-associated repressor of the glucocorticoid receptor. Sci Signal 3: ra8, 2010.

69. Chen L, Ren P, Zhang Y, Gong B, Yu D and Sun X: Long non-coding RNA GAS5 increases the radiosensitivity of A549 cells through interaction with the miR-21/PTEN/Akt axis. Oncol Rep 43: 897-907, 2020.

70. Dong Z, Li S, Wang X, Si L, Ma R, Bao L and Bo A: lncRNA GAS5 restrains $\mathrm{CCl}_{4}$-induced hepatic fibrosis by targeting miR-23a through the PTEN/PI3K/Akt signaling pathway. Am J Physiol Gastrointest Liver Physiol 316: G539-G550, 2019.

71. Shen Z and She Q: Association between the deletion allele of Ins/Del polymorphism (Rs145204276) in the promoter region of GAS5 with the risk of atherosclerosis. Cell Physiol Biochem 49: 1431-1443, 2018

72. Zhu L, Zhu Q, Wen H, Huang X and Zheng G: Mutations in GAS5 affect the transformation from benign prostate proliferation to aggressive prostate cancer by affecting the transcription efficiency of GAS5. J Cell Physiol 234: 8928-8940, 2019. 
73. Liu K, Liu C and Zhang Z: lncRNA GAS5 acts as a ceRNA for miR-21 in suppressing PDGF-bb-induced proliferation and migration in vascular smooth muscle cells. J Cell Biochem 120: 15233-15240, 2019.

74. Zhou XH, Chai HX, Bai M and Zhang Z: IncRNA-GAS5 regulates PDCD4 expression and mediates myocardial infarction-induced cardiomyocytes apoptosis via targeting MiR-21. Cell Cycle 19: $1363-1377,2020$

75. Zheng D, Hou Y,Li Y, Bian Y, Khan M, Li F, Huang L and Qiao C: Long non-coding RNA Gas5 is associated with preeclampsia and regulates biological behaviors of trophoblast via MicroRNA-21. Front Genet 11: 188, 2020.

76. Han Y, Wu N, Xia F, Liu S and Jia D: Long non-coding RNA GAS5 regulates myocardial ischemia-reperfusion injury through the PI3K/AKT apoptosis pathway by sponging miR-532-5p. Int J Mol Med 45: 858-872, 2020.
77. $\mathrm{Li} \mathrm{J}, \mathrm{Lv} \mathrm{H}$ and Che YQ: Long non-coding RNA Gas5 potentiates the effects of microRNA-21 Downregulation in response to ischaemic brain injury. Neuroscience 437: 87-97, 2020.

78. Wu N, Zhang X, Bao Y, Yu H, Jia D and Ma C: Down-regulation of GAS5 ameliorates myocardial ischaemia/reperfusion injury via the miR-335/ROCK1/AKT/GSK-3 $\beta$ axis. J Cell Mol Med 23: 8420-8431, 2019.

79. He J, Dai H, Zhao Q, Guo J and Chen C: LNCRNA Gas5 suppression protected $\mathrm{Hl}-1$ cells against hypoxia injury by sponging miR-222-3p. Exp Mol Pathol 115: 104436, 2020.

(7)(9) This work is licensed under a Creative Common EY No No Attribution-NonCommercial-NoDerivatives 4.0 International (CC BY-NC-ND 4.0) License. 